\title{
ON THE THEORY OF RING-LOGICS
}

\author{
ADIL YAQUB
}

Introduction. Boolean rings $(B, \times,+)$ and Boolean logics (= Boolean algebras) $(B, \cap, *)$ are equationally interdefinable in a familiar way (6). Foster's theory of ring-logics $(\mathbf{1} ; \mathbf{2} ; \mathbf{3})$ raises this interdefinability and indeed the entire Boolean theory to a more general level. In this theory a ring (or an algebra) $R$ is studied modulo $K$, where $K$ is an arbitrary transformation group (or "Coordinate transformations") in $R$. The Boolean theory results from the special choice, for $K$, of the "Boolean group," generated by $x^{*}=1-x$ (order $\left.2, x^{* *}=x\right)$. More generally, in a commutative ring $(R, \mathrm{X},+)$ with identity the natural group $N$, generated by $x^{\Lambda}=1+x$ (with $x^{\mathbf{v}}=x-1$ as inverse) was shown to be of particular interest. Thus specialized to $N$, a commutative ring with identity $(R, \times,+)$ is called a ring-logic, $\bmod N$, if (1) the + of the ring is equationally definable in terms of its $N$-logic $(R$, $\left.\mathrm{X},{ }^{\boldsymbol{\Lambda}}, \mathrm{v}\right)$, and (2) the + of the ring is fixed by its $N$-logic. It was shown (2) that each $p$-ring (5) is a ring-logic $\bmod N$. It was further shown (3) that each $p^{k}$-ring $(3 ; 5)$ is a ring-logic $\bmod D$, where $D$ is a somewhat more involved group.

All these known examples of ring-logics have zero radical, and the question presents itself: do there exist examples of ring-logics (modulo a suitable group) with non-zero radical? We shall answer this in the affirmative. Indeed, we shall show that the ring of residues mod $n$ ( $n$ arbitrary) is a ring-logic modulo the natural group $N$ itself.

1. The ring of residues $\bmod p^{k}$. Let $(R, \times,+)$ be a commutative ring with identity 1 . We denote the generator of the natural group $N$ by

$$
x^{\Lambda}=1+x,
$$

with inverse

$$
x^{\mathrm{v}}=x-1
$$

As in (1), we define

$$
a \times_{\Lambda} b=\left(a^{\Lambda} \times b^{\Lambda}\right)^{\mathbf{v}}
$$

It is readily verified that

$$
a \times_{\mathrm{A}} b=a+b+a b .
$$

The following notation is used (2):

$$
x^{\Lambda_{n}}=\left(\ldots\left(\left(x^{\Lambda}\right)^{\Lambda}\right) \ldots\right)^{\Lambda} ; \quad x^{v_{n}}=\left(\ldots\left(\left(x^{v}\right)^{v}\right) \ldots\right)^{\mathbf{v}}
$$

$n$ iterations. Again

$$
x^{\Lambda_{k} n}=\left(x^{\Lambda_{k}}\right)^{n} ; \quad x^{\mathrm{v} k n}=\left(x^{\mathrm{v} k}\right)^{n} .
$$

Received September 9, 1955. 
We now consider $\left(R_{p^{k}}, \times,+\right)$, the ring of residues $\bmod p^{k}(p$ prime) and prove the following

TheOREM 1. $\left(R_{p^{k}}, \times,+\right)$ is a ring-logic $(\bmod N)$. The ring + is given by the following $N$-logical formula

$$
\begin{aligned}
x+y=\left\{\left(x\left(y x^{p^{k}-p^{k-1}-1}\right)^{\Lambda}\right)\right. & \left.x^{p^{k}-p^{k-1}}\right\} \times_{\Lambda} \\
& \left\{\left(x^{\Lambda}\left(y\left(x^{\Lambda}\right)^{p^{k}-p^{k-1}-1}\right)^{\Lambda}\right)^{\mathrm{v}}\left(x^{p^{k}-p^{k-1}}\right)^{\mathrm{v} 2}\right\} .
\end{aligned}
$$

Proof. By Euler's generalized form of Fermat's Theorem, we have

$$
a^{p^{k}-p^{k-1}}=1, a \in R_{p^{k}}
$$

$a$ not divisible by $p$. We now distinguish two cases:

Case 1: Suppose $p$ does not divide $x$. Then, by (1.6), the right side of (1.5) reduces to

$$
\left\{x\left(1+y x^{p^{k}-p^{k-1}-1}\right) \cdot 1\right\} \times_{\Lambda} 0=x+y x^{p^{k}-p^{k-1}}=x+y,
$$

since

$$
\left(x^{p^{k}-p^{k-1}}\right)^{\mathrm{v} 2}=1^{\mathrm{v} 2}=0 ; a \times_{\Lambda} 0=a .
$$

This proves (1.5).

Case 2: Suppose $p$ divides $x$. Then, clearly, $p$ does not divide $x^{\Lambda}=1+x$. Hence, using Case 1 , the right side of (1.5) reduces to

$$
\begin{aligned}
0 \times_{\Lambda}\left\{\left(x^{\Lambda}\left(1+y\left(x^{\Lambda}\right)^{p^{k}-p^{k-1}-1}\right)\right)^{\mathbf{v}} \cdot 1\right\} & =\left(x^{\Lambda}+y\left(x^{\Lambda}\right)^{p^{k}-p^{k-1}}\right)^{\mathbf{v}} \\
& =\left(x^{\Lambda}+y\right)^{\mathbf{v}}=x+y
\end{aligned}
$$

since

$$
\left(x^{p^{k}-p^{k-1}}\right)^{\mathrm{v} 2}=0^{\mathrm{v} 2}=1 ; 0 \times_{\Lambda} a=a .
$$

Again (1.5) is verified. Hence $\left(R_{p^{k}}, X,+\right)$ is equationally definable in terms of its $N$-logic. Next, we show that $\left(R_{p k}, \mathrm{X},+\right)$ is fixed by its $N$-logic. ${ }^{1}$ Suppose then that there exists another ring $\left(R_{p^{k}}, \times,+^{\prime}\right)$, with the same class of elements $R_{p^{k}}$ and the same $\times$ as $\left(R_{p^{k}}, \times,+\right)$ and which has the same logic as $\left(R_{p^{k}}, \times,+\right)$. To prove that $+=+^{\prime}$. Again we distinguish two cases.

Case 1: $p$ does not divide $x$. Then

$$
x+^{\prime} y=x\left(1+{ }^{\prime} y x^{p^{k}-p^{k-1}-1}\right)=x\left(y x^{p^{k}-p^{k-1}-1}\right)^{\Lambda}=x\left(1+y x^{p^{k}-p^{k-1}-1}\right)=x+y,
$$
since, by hypothesis, $x^{\Lambda}=1+x=1+^{\prime} x$.

${ }^{1} \mathrm{~A}$ ring $(R, \times,+)$ is said to be fixed by its $N$-logic if there exists no other ring $\left(R, \times,+^{\prime}\right)$, on the same set $R$ and with the same $\times$ but with $+^{\prime} \neq+$, which has the same $N$-logic; i.e.,

$$
x^{\wedge}=1+x=1+^{\prime} x ; x^{\mathrm{v}}=x-1=x-^{\prime} 1 .
$$


Case 2: $p$ divides $x$. Then, clearly, $p$ does not divide $x^{\Lambda}=1+x$. Hence, by Case 1,

$$
x+^{\prime} y=x^{\Lambda}+^{\prime} y^{\mathrm{v}}=x^{\Lambda}+y^{\mathrm{v}}=x+y .
$$

Therefore $+^{\prime}=+$, and the theorem is proved.

Corollary. $\left(R_{p}, \times,+\right)=\left(F_{p}, \times,+\right)$, the ring (field) of residues $(\bmod p)$, $p$ prime, is a ring-logic ( $\bmod N)$ the + being given by setting $k=1$ in (1.5), and making use of $x^{p}=x$ :

$$
x+y=\left\{\left(x\left(x^{p-2} y\right)^{\Lambda}\right)\right\} \times_{\Lambda}\left\{\left(x^{\Lambda}\left(\left(x^{\Lambda}\right)^{p-2} y\right)^{\Lambda}\right)^{\mathrm{v}}\left(x^{p-1}\right)^{\mathrm{v} 2}\right\} .
$$

2. The ring of residues $(\bmod n), n$ arbitrary. In attempting to generalize Theorem 1 to the residue class ring $\left(R_{n}, \mathrm{X},+\right)$, where $n$ is any positive integer, the following concept of independence, introduced by Foster (4), is needed.

Definition. Let $\mathfrak{\mathfrak { I }}=\left\{\mathfrak{A}_{1}, \mathfrak{A}_{2}, \ldots, \mathfrak{A}_{n}\right\}$ be a finite set of algebras of the same species $\mathfrak{\subseteq}$. We say that the algebras $\mathfrak{A}_{1}, \mathfrak{A}_{2}, \ldots, \mathfrak{A}_{n}$ are independent if, corresponding to each set $\left\{\phi_{i}\right\}$ of expressions of species $\subseteq(i=1, \ldots, n)$, there exists at least one expression $X$ such that

$$
\phi_{i}=X\left(\bmod \mathfrak{A}_{i}\right) \quad(i=1, \ldots, n) .
$$

By an expression we mean some composition of one or more indeterminatesymbols $\zeta, \ldots$ in terms of the primitive operations of $\mathfrak{A}_{1}, \mathfrak{A}_{2}, \ldots, \mathfrak{A}_{n} ; \phi=X$ $(\bmod \mathfrak{U})$, also written as $\phi=X(\mathfrak{U})$, means that this is an identity of the algebra $\mathfrak{A}$.

We now prove the following

TheOREM 2. Let $\left(\mathfrak{A}_{1}, \times,+\right), \ldots,\left(\mathfrak{A}_{t}, \times,+\right)$ be a finite set of ring-logics $(\bmod N)$, such that the $N$-logics $\left(\mathfrak{A}_{1}, \times{ }^{\wedge}\right), \ldots,\left(\mathfrak{A}_{t}, \times,^{\wedge}\right)$ are independent. Then $\mathfrak{A}=\mathfrak{A}_{1} \times \ldots \times \mathfrak{A}_{\imath}($ direct product $)$ is also a ring-logic $(\bmod N)$.

Proof. Since $\mathfrak{A}_{i}$ is a ring-logic $(\bmod N)$, there exists an $N$-logical expression $\phi_{i}$ such that, for every $x_{i}, y_{i} \in \mathfrak{A}_{i}(i=1, \ldots, t)$,

$$
x_{i}+y_{i}=\phi_{i}=\phi_{i}\left(x_{i}, y_{i} ; \times,^{\Lambda},{ }^{\mathrm{v}}\right)=\phi_{i}\left(x_{i}, y_{i} ; \times,^{\Lambda}\right) \text {. }
$$

In view of the independence of the logics, there exists an expression $X$ such that

$$
X=\left\{\begin{array}{l}
\phi_{1}\left(\bmod \mathfrak{U}_{1}\right), \\
\cdots \\
\phi_{t}\left(\bmod \mathfrak{U}_{t}\right) .
\end{array}\right.
$$

Then, for $a=\left(a_{1}, a_{2}, \ldots, a_{t}\right) \in \mathfrak{A} ; b=\left(b_{1}, b_{2}, \ldots, b_{t}\right) \in \mathfrak{A}$, we have

\footnotetext{
${ }^{2}$ This formula is considerably shorter than the formulas for + given in $(2 ; 3)$.
} 


$$
\begin{aligned}
X\left(a, b ; \times,{ }^{\Lambda}\right) & =X\left(\left(a_{1}, a_{2}, \ldots, a_{t}\right),\left(b_{1}, b_{2}, \ldots, b_{t}\right) ; \times,{ }^{\Lambda}\right) \\
& =\left(X\left(a_{1}, b_{1} ; \times, \Lambda\right), X\left(a_{2}, b_{2} ; \times, \Lambda\right), \ldots, X\left(a_{t}, b_{t} ; \times,^{\Lambda}\right)\right) \\
& =\left(a_{1}+b_{1}, a_{2}+b_{2}, \ldots, a_{t}+b_{t}\right) \\
& =\left(a_{1}, a_{2}, \ldots, a_{t}\right)+\left(b_{1}, b_{2}, \ldots, b_{t}\right) \\
& =a+b
\end{aligned}
$$

i.e.,

$$
a+b=X\left(a, b ; \times{ }^{\wedge}\right) ; a, b \in \mathfrak{A} .
$$

Hence, $\mathfrak{A}=\mathfrak{A}_{1} \times \ldots \times \mathfrak{A}_{t}$ is equationally definable in terms of its $N$-logic. Next, we show that $\mathfrak{A}$ is fixed by its $N$-logic. Suppose there exists a $+^{\prime}$ such that $\left(\mathfrak{A}, \times,+^{\prime}\right)$ is a ring, with the same class of elements $\mathfrak{A}$ and the same $X$ as the ring ( $\mathfrak{A}, X,+)$, and which has the same logic $\left(\mathfrak{A}, X^{\wedge}\right)$ as the ring $(\mathfrak{A}, \times,+)$. To prove that $+=+^{\prime}$.

Now, let $a=\left(a_{1}, a_{2}, \ldots, a_{t}\right) \in \mathfrak{A} ; b=\left(b_{1}, b_{2}, \ldots, b_{t}\right) \in \mathfrak{A}$. A new $+^{\prime}$ in $\mathfrak{A}$ defines and is defined by new $+^{\prime}{ }_{1}$ in $\mathfrak{I}_{1},+^{\prime}{ }_{2}$ in $\mathfrak{A}_{2}, \ldots,+{ }^{\prime}{ }_{t}$ in $\mathfrak{A}_{t}$, such that $\left(\mathfrak{U}_{1}, X,+^{\prime}{ }_{1}\right)$ is a ring, and similarly for $\left(\mathfrak{U}_{2}, X,+^{\prime}{ }_{2}\right), \ldots,\left(\mathfrak{A}_{l}, X,+^{\prime}{ }_{t}\right)$; i.e.,

$$
\begin{aligned}
a+{ }^{\prime} b & =\left(a_{1}, a_{2}, \ldots, a_{t}\right)+{ }^{\prime}\left(b_{1}, b_{2}, \ldots, b_{t}\right) \\
& =\left(a_{1}+{ }^{\prime}{ }_{1} b_{1}, a_{2}+{ }^{\prime} b_{2}, \ldots, a_{t}+{ }^{\prime}{ }_{t} b_{t}\right) .
\end{aligned}
$$

Furthermore, the assumption that $\left(\mathfrak{A}, \times,+^{\prime}\right)$ has the same logic as $(\mathfrak{A}, \times,+)$ is equivalent to the assumption that $\left(\mathfrak{A}_{1}, X,+^{\prime}{ }_{1}\right)$ has the same logic as $\left(\mathfrak{A}_{1}, \times,+\right)$, and similarly for $\left(\mathfrak{A}_{i}, \times,+^{\prime}{ }_{i}\right)$ and $\left(\mathfrak{A}_{i}, \times,+\right)(i=2, \ldots, t)$. Since $\left(\mathfrak{A}_{1}, X,+\right)$ is a ring-logic, and hence with its + fixed, it follows that $+^{\prime}{ }_{1}=+$; similarly $+^{\prime}{ }_{2}=+, \ldots,+^{\prime}{ }_{t}=+$. Hence, using $(2.1),+^{\prime}=+$, and the proof is complete.

We shall now prove the following

LeMma 3. Let $p_{1}, \ldots, p_{t}$ be distinct primes, and let

$$
\left(R_{n i}, \times,+\right), n_{i}=p_{i}^{k_{i}}=p_{i} m_{i} ; i=1, \ldots, t,
$$

be a set of residue class rings $\left(\bmod n_{i}\right)$. Then the logics $\left(R_{n_{i}}, \times,{ }^{\Delta}\right)(i=1, \ldots, t)$ are independent.

Proof. Let

$$
P(i)=\prod_{j=1}^{t} n_{j}, \quad j \neq i
$$

Then, clearly

$$
\left(P(i), n_{i}\right)=1 \text {. }
$$

Hence, there exist integers $r_{i}>0, s_{i}>0$ such that

$$
r_{i} P(i)-s_{i} n_{i}=1 \text {. }
$$

Now, define

$$
\epsilon(x)=x^{\left(n_{1}-m_{1}\right)\left(n_{2}-m_{2}\right) \ldots\left(n_{t}-m_{t}\right)} .
$$


Then one easily verifies that, for $i \neq j$,

$$
\omega_{i}=\omega_{i}(x)=\left\{\epsilon(x) \times_{\Lambda}\left((\epsilon(x))^{\mathrm{V}}\right)^{\left(n_{1}-m_{1}\right) \ldots\left(n_{t}-m_{t}\right)}\right\}^{\Lambda_{r_{i}} P(i)-1}=\left\{\begin{array}{l}
1\left(R_{n_{i}}\right) \\
0\left(R_{n_{j}}\right)
\end{array} .\right.
$$

Now, to prove the independence of the logics $\left(R_{n_{i}}, \times,{ }^{\wedge}\right)$, let $\left\{\phi_{i}\right\}$ be a set of $t$ expressions of species $\times{ }^{\wedge}$; i.e., a primitive composition of indeterminatesymbols in terms of the operations $X,{ }^{\wedge}$; then, if we define (cf. 4)

$$
X=\phi_{1} \omega_{1} \times_{\Lambda} \phi_{2} \omega_{2} \times_{\Lambda} \ldots \times_{\Lambda} \phi_{t} \omega_{t},
$$

we immediately obtain

$$
\phi_{i}=X\left(\bmod R_{n_{i}}\right),
$$

since $a \times{ }_{\Lambda} 0=a=0 \times{ }_{\Lambda} a$. This proves the theorem.

Recalling the well-known fact that

$$
\left(R_{n}, \times,+\right) \cong R_{n_{1}} \times \ldots \times R_{n t} \text { (direct product), }
$$

$n$ arbitrary, $n=n_{1} \ldots n_{t}$, a combination of Theorems 1, 2, Lemma 3 and (2.2) readily yields

Theorem 4 (Fundamental Theorem on $R_{n}$ as ring-logics). $\left(R_{n}, \mathrm{X},+\right)$, the residue class ring $(\bmod n), n$ arbitrary, is a ring-logic $(\bmod N)$.

We conclude with several illustrative examples.

Example 1. $R_{p^{k}}=R_{2}=F_{2}=\{0,1\}$.

It is readily verified that each of (1.5) and (1.7) reduces to the familiar Boolean formula

$$
x+y=x y^{\Lambda} \times_{\Lambda} x^{\Lambda} y .
$$

Example 2. $R_{p^{k}}=R_{3}=F_{3}=\{0,1,2\}$.

Formula (1.7) yields

$$
x+y=\left\{x(x y)^{\wedge}\right\} \times_{\Lambda}\left\{\left[\left(x^{\Lambda}\left(x^{\wedge} y\right)^{\Lambda}\right)\right]^{\mathrm{v}}\left(x^{2}\right)^{\mathbf{v} 2}\right\} .
$$

Compare with (1) in which the following formula was obtained:

$$
x+y=x y^{\wedge}{ }_{\Lambda} x^{\wedge} y \times{ }_{\Lambda} x^{2} y^{2} .
$$

It is noteworthy to observe that the + of $\left(F_{p}, \times,+\right)$, the field of residues $(\bmod p), p$ prime, may also be expressed in the following form:

$$
x+y=\left\{x\left(y x^{p-2}\right)^{\Lambda}\right\} \times_{\Lambda}\left\{y\left(x^{\Lambda} x^{\Lambda} \ldots x^{\Lambda_{p-1}}\right)^{2}\right\} .
$$

or by

$$
x+y=\left\{x\left(y x^{p-2}\right)^{\wedge}\right\} \times_{\mathbf{A}}\left\{y\left(x^{p-1}\right)^{\mathbf{v} 2}\right\} .
$$

The last formula, when specialized to $F_{3}$, gives a simpler expression for + than (2.4). 
Example 3. $R_{p^{k}}=R_{2^{2}}=\{0,1,2,3\}$.

Formula (1.5) reduces to

$$
x+y=\left\{\left(x(x y)^{\Lambda} x^{2}\right)\right\} \times_{\Lambda}\left\{\left[\left(x^{\Lambda}\left(x^{\Lambda} y\right)^{\Lambda}\right)\right]^{\mathrm{v}}\left(x^{2}\right)^{\mathrm{v} 2}\right\} .
$$

It may be verified that the + in $\left(R_{4}, X,+\right)$ is also given by

$$
x+y=\left\{(x y)^{\Lambda}(x y)^{2} \times_{\Lambda}\left(x \times_{\Lambda} y\right)(x y)^{\Lambda 2}\right\}\left\{(x y)(x y)^{2 \mathrm{v}}\right\}^{\Lambda} .
$$

This last formula excels most of the others in obviously displaying the symmetry of + .

Example 4. $R_{n}=R_{6}=\{0,1,2,3,4,5\}$.

The correspondence

$$
\begin{array}{ll}
0 \leftrightarrow\left(0_{2}, 0_{3}\right), & 3 \leftrightarrow\left(1_{2}, 0_{3}\right), \\
1 \leftrightarrow\left(1_{2}, 1_{3}\right), & 4 \leftrightarrow\left(0_{2}, 1_{3}\right), \\
2 \leftrightarrow\left(0_{2}, 2_{3}\right), & 5 \leftrightarrow\left(1_{2}, 2_{3}\right),
\end{array}
$$

determines an isomorphism of $R_{6}$ and $R_{2} \times R_{3}$ (direct product), where $R_{2}=$ $\left\{0_{2}, 1_{2}\right\}$ and $R_{3}=\left\{0_{3}, 1_{3}, 2_{3}\right\}$.

It is readily verified (compare with the proof of Lemma 3 and (2.3), (2.5) above) that

$$
\text { (2.10) } \begin{aligned}
x+y=\left\{\left(x y^{\Lambda} \times_{\Delta} x^{\Lambda} y\right)\right. & \left.\left(x^{2} \times_{\Lambda}\left(x^{2}\right)^{\mathrm{v} 2}\right)^{\Lambda_{2}}\right\} \\
& \times_{\Lambda}\left\{\left(x y^{\Lambda} \times_{\Lambda} x^{\Lambda} y \times_{\Delta} x^{2} y^{2}\right)\left(x^{2} \times_{\Lambda}\left(x^{2}\right)^{\mathrm{v} 2}\right)^{\Lambda_{3}}\right\} .
\end{aligned}
$$

Formula (2.10) may be verified either by direct substitution from $R_{6}$, or via the $R_{2} \times R_{3}$ representation above.

\section{REFERENCES}

1. A. L. Foster, On n-ality theories in rings and their logical algebras, including tri-ality principle in three-valued logics, Amer. J. Math., 72 (1950), 101-123.

2. - - p-rings and ring-logics, Univ. Calif. Publ., 1 (1951), 385-396.

3. - - $p^{k}$-rings and ring-logics, Ann. Scu. Norm. Pisa, 5 (1951), 279-300.

4. - Unique subdirect factorization within certain classes of universal algebras, Math. Z., 62 (1955), 171-188.

5. N. H. McCoy and D. Montgomery, A representation of generalized Boolean rings, Duke Math. J., 3 (1937), 455-459.

6. M. H. Stone, The theory of representations of Boolean algebras, Trans. Amer. Math. Soc., 40 (1936), 37-111.

University of California, Berkeley

and

Purdue University 